choice of $\epsilon$. The usual procedure is to divide the range of integration into two parts, integrate outwards for a solution satisfying one boundary condition, integrate inwards for a solution satisfying the other boundary condition, match the solutions at an intermediate point and adjust $\epsilon$ so that the derivatives also agree [1], [2]. The inward integration may be avoided with the procedure described earlier. A convenient way of dividing the range is according to the sign of $f(r)$. For some $r$, $f(r)<0$ so that condition (ii) is not satisfied: the procedure described here is not always numerically stable when $f(r)<0$ [3]; in fact, for some values of $i,\left|d_{i}\right|<1$. Of a series of standard methods, the Numerov method,

$$
\begin{aligned}
y_{n+1}=\left(\left(2+\frac{10}{12} h^{2} f_{n}\right) y_{n}\right. & -\left(1-\frac{h^{2}}{12} f_{n-1}\right) y_{n-1} \\
& \left.+\frac{h^{2}}{12}\left(g_{n+1}+10 g_{n}+g_{n-1}\right)\right) /\left(1-\frac{h^{2}}{12} f_{n+1}\right),
\end{aligned}
$$

was found to be most accurate in this case, for a given number of evaluations of $f$ for the outward integration. The procedure used successfully was to integrate outwards according to (5) until $f(r)>0$, then, with the last value computed as a boundary condition, to solve for the "tail" of the wave function by the method described here. The energy adjustment will be the same as before.

Department of Mathematics

University of British Columbia

Vancouver, B. C.

1. J. W. Cooley, "An improved eigenvalue corrector formula for solving the Schrödinger equation for central fields," Math. Comp., v. 15, 1961, p. 363-374.

2. A. S. Douglas, "On the Sturm-Liouville equation with two-point boundary conditions," Proc. Cambridge Philos. Soc., v. 52, 1956, p. 636-639.

3. E. C. RIDLEY, "A numerical method of solving second-order linear differential equations with two-point boundary conditions," Proc. Cambridge Philos. Soc., v. 53, 1957, p. 442-447.

\title{
On the Inversion of Sparse Matrices
}

\section{By A. L. Dulmage and N. S. Mendelsohn}

1. Introduction. There are a number of problems in applied mathematics involving many equations in many unknowns, but for which each equation involves only a small fraction of the unknowns. If such problems are linear or are approximated by linearization, one is involved with a matrix, a large proportion of whose entries are zero. To invert such a matrix $A$ it is sometimes advantageous to permute the rows and columns of $A$ yielding $P A Q$ where $P$ and $Q$ are permutation matrices. If

$$
P A Q=\left|\begin{array}{ccc}
A_{1} & & 0 \\
A_{2} & \\
& \ddots & \\
* & & A_{r}
\end{array}\right|
$$

where $A_{1}, A_{2}, \cdots A_{r}$ are square matrices, the problem of inverting $P A Q$ is reduced

Received September 18, 1961. This research was supported by the United States Air Force Office of Scientific Research. 
to inverting the blocks $A_{r}$ followed by matrix multiplication and addition. On permuting the rows and columns of $(P A Q)^{-1}$ one obtains the matrix $A^{-1}$.

F. Harary [4] gave a method based on the connectivity theory of directed graphs. The blocks $A_{i}$ turn out to be the matrix representatives of the strong components of an associated directed graph. Harary's method requires that $Q=P^{-1}$, a restriction which is quite unnecessary for matrix inversion. As a result, many matrices which reduce under independent permutations of rows and columns will not reduce if one insists that $Q=P^{-1}$. To remove this latter restriction, the authors have replaced a directed graph by a bipartite graph. The strong components of a directed graph become the irreducible components of a bipartite graph (see [1] for the definition of an irreducible component of a bipartite graph, [2] for the connection between strong components of directed graphs and irreducible components of bipartite graphs).

2. The Method of Reduction. Let $A$ be a square matrix of order $n$ with entries $a_{i j}$. Associated with $A$ is a bipartite graph $K_{A}$ with two sets of vertices $S=s_{1}$, $s_{2}, \cdots, s_{n}$ and $T=t_{1}, t_{2}, \cdots, t_{n}$. A pair $\left(s_{i}, t_{j}\right)$ is an edge of $K_{A}$ if and only if $a_{i j} \neq 0$ (one obtains Harary's directed graph if one identifies the vertices $s_{i}$ and $\left.t_{i}\right)$.

Suppose we can find matrices $P$ and $Q$ such that

$$
P A Q=\left|\begin{array}{ccc}
A_{1} & & 0 \\
A_{2} & & \\
& \ddots & \\
* & & A_{r}
\end{array}\right|
$$

and such that $A_{1}, A_{2}, \cdots, A_{r}$ cannot be further reduced by permutations of their rows and columns. Then the graphs corresponding to $A_{1}, A_{2}, \cdots, A_{r}$ are the irreducible components of $K_{A}$.

In [3], the authors give an algorithm for obtaining the irreducible components of a bipartite graph. This algorithm is easily programmable for machine computation and yields the permutations $P$ and $Q$. In the case where $A$ is a non-singular matrix the graph $K_{A}$ has no tails (see [3] for definition of a tail) and the algorithm described in [3] can be considerably simplified, since the steps needed to isolate and identify the tails can be omitted. An alternative procedure is the following. First, locate a nonzero term in the expansion of the determinant of $A$. This can readily be done using the algorithm of Marshall Hall [5] or Fulkerson and Ford [6]. Next, permute the rows of $A$ until the entries of this term occupy the main diagonal. Call this new matrix $A^{*}$. Finally, apply the method of F. Harary given in [4] to $A^{*}$.

We append an example:

$$
\text { Let } A=\left|\begin{array}{lllllll}
0 & 0 & 5 & 0 & 0 & 1 & 0 \\
0 & 2 & 0 & 0 & 0 & 0 & 4 \\
1 & 0 & 0 & 2 & 0 & 0 & 0 \\
0 & 4 & 0 & 3 & 0 & 0 & 0 \\
3 & 0 & 1 & 0 & 3 & 2 & 0 \\
3 & 2 & 0 & 0 & 2 & 0 & 0 \\
1 & 0 & 0 & 4 & 0 & 0 & 0
\end{array}\right|
$$


Note that the corresponding directed graph has the Hamiltonian circuit $1 \rightarrow 6 \rightarrow$ $5 \rightarrow 3 \rightarrow 4 \rightarrow 2 \rightarrow 7 \rightarrow 1$ and so is strongly connected. Hence, for no permutation $P$ does $P A P^{-1}$ reduce. Using the algorithm described in [3] one obtains the permutations $P=(1725643$ ) and $Q=(1)(236754)$. Applying $P$ and $Q$ to the rows and columns of $A$, one obtains:

$$
P A Q=\left|\begin{array}{cc:ccc:cc}
1 & 2 & 0 & 0 & 0 & 0 & 0 \\
1 & 4 & 0 & 0 & 0 & 0 & 0 \\
\hdashline 0 & 3 & 4 & 0 & 0 & 0 & 0 \\
3 & 0 & 2 & 2 & 0 & 0 & 0 \\
0 & 0 & 2 & 0 & 4 & 0 & 0 \\
\hdashline 3 & 0 & 0 & 3 & 0 & 1 & 2 \\
0 & 0 & 0 & 0 & 0 & 5 & 1
\end{array}\right|
$$

The authors are indebted to F. Harary for a pre-publication copy of his paper [4].

The University of Manitoba

Winnipeg, Canada

1. A. L. Dulmage \& N. S. Mendelsohn, "A structure theory of bipartite graphs of finite exterior dimension," Trans. Roy. Soc. Canada, Sect. III 53, 1959, p. 1-13.

2. D. M. Johnson, A. L. Dulmage, \& N. S. Mendelsohn, "Connectivity and reducibility of graphs", Canad.J. Math., 14, 1962, p. 529-539.

3. A. L. Dulmage \& N. S. Mendelsohn, "Two algorithms for bipartite graphs," to be published in Soc. Indust. Appl. Math.

4. F. HARARY, "A graph theoretic approach to matrix inversion by partitioning," to be published in Numer. Math.

5. M. HALL, "An algorithm for distinct representatives," Amer. Math. Monthly, 1956, p. $716-717$.

6. L. R. Ford \& D. R. Fulkerson, "A simple algorithm for finding maximal network flows and an application to the Hitchcock problem," Canad.J. Math. 9, 1957, p. 210-218. Also in Management Sci., October 1956, p. 24-32.

\section{Missing Data Correlation Computations}

\section{By R. I. Jennrich}

In correlation analysis or in any multivariate analysis based on the computation of a correlation or covariance matrix, the applied statistician often runs into the problem of missing data. To avoid complication in computing the correlation matrix, a complete observation vector is often discarded when only one or more of its components are missing. If a correlation matrix is computed by means of a standard electronic computer program, this procedure is often necessary. A large percentage of data may be thrown away when only a small percentage is missing. This note describes a modification in the standard computing scheme which eliminates this waste of data.

Let $x_{n 1}, x_{n 2}, \cdots, x_{n p}$ denote the $p$ components of the $n$th observation vector, $n=1,2, \cdots, N$. It is customary to add an $n+1$ st component to this vector which is identically equal to one. That is $x_{n, p+1}=1$. The cross product matrix

$$
a_{i j}=\sum_{n=1}^{N} x_{n i} x_{n j} \quad i=1, \cdots, p+1 ; j=1, \cdots, p+1
$$

Received September 7, 1961. 